\title{
ENDO- AND EXO-THEORIES OF MATTER
}

\author{
HANS PRIMAS \\ Laboratory of Physical Chemistry, ETH \\ ETH-Zentrum, CH-8092 Zürich, Switzerland
}

\begin{abstract}
The endo/exo-dichotomy in the domain of the material world is analyzed from the standpoint of old-fashioned Cartesian dualism. The holistic structure of the quantum world enforces a Platonic view for the universally valid laws governing matter. In contrast to the structural simplicity of non-operational quantum endophysics, the empirical reality and experiments belong to quantum exophysics. In algebraic quantum mechanics, the transition from quantum endophysics to quantum exophysics is mathematically welldefined. The exophysical richness and variety of the concrete and particular emerges by imposing a new, contextually selected topology into fundamental endophysics. In the Cartesian world view, the inverse problem, however, is ill-posed. Though the endophysical realm can be grasped by human reason, endophysics cannot be set up from sense data and experiments without recourse to primordial images.
\end{abstract}

\section{Endo- and exo-description in the material domain}

A pertinent distinction referring to internal vs. external viewpoints has been introduced by Otto Rössler [34], and David Finkelstein [10,11] under the name «endophysics» and «exophysics», respectively. In spite of the fact that this endo/exo distinction is of utmost importance, there is not yet a general agreement about the essence of this new notion. The reader should therefore be aware that in spite of similar (but not quite identical) definitions, various authors associate quite different ideas and research programs with this endo/exodichotomy. I will adopt the following characterization ${ }^{1}$ :

\section{Definition 1: A system without accessible external observers is called an endosystem.}

Definition 2: A part of the world which is split into an observed system and an observing system is called a universe of discourse.

Definition 3: The tools of observation and communication (which may or may not include human observers) constitute an exosystem.

Definition 4: The system observed is called object.

Definition 5: A metatheoretical distinction generating an object and an exosystem from an endosystem is called a cut.

1 This formulation is inspired by the discussions we had at the Ringberg Workshop, and is based in an essential way on a proposal by Dr. Eva Ruhnau. These definitions are more general but compatible with those used in [26-31] and [33]. Note that Finkelstein's definition of an endosystem is different [10-12]. Finkelstein writes: "In general, quantum theory asserts that the experimenter and the experimentee are parts of an inseparable whole system, insists that nevertheless we must partition this system, and does not tell us how. Call the two parts of this partition the endosystem and exosystem; the endosystem is entity under study, and the exosystem includes the experimental apparatus, and the relevant external environment." [12]. In my terminology, this setting is a universe of discourse, giving an exophysical description of an open object (Finkelstein's endosystem) with a Heisenberg cut between the object system and the measuring tools. That is, only for the exosystem we have the same terminology. 
Inescapably, the study of the endo/exo-dichotomy must be conducted in a metalanguage. The language in which any endo/exo-theory can ever be formulated is neither part of the endosystem nor of the exosystem.

I will consider here neither the complementarity of matter and mind nor my possibility to know my own body from without - as an object among other objects -, and from within as a center of my action. Since the endo/exo-dichotomy is of importance already in the realm of traditional physics, I think it is a reasonable idea at first to restrict the discussion to purely material systems, a domain where contemporary science provides powerful mathematical tools for a study of this difficult problem.

For a scientific description of the material reality it is inevitable to distinguish between spirit and matter. Historically, this enforced separation was crucial for the development of an objectivistic science. It was René Descartes, who first articulated clearly the idea of an objective external world, separate and radically distinct from the human mind. The Cartesian duality of mind and body, the mind being immaterial substance (res cogitans, «thinking substance»), and the body being material substance (res extensa, «extended substance») was the basis for the development of the modern scientific world view. In contemporary science, spirit and matter are hopelessly split into two irreconcilable halves. Of course, there are grave and often discussed problems with the Cartesian dualism of mind and body. From the point of view of experimental science, the fundamental issue of the mind-body problem is the question of free will. It is a tacit assumption of all engineering sciences that nature can be manipulated and that proper initial conditions can be brought about by interventions of the world external to the object under investigation. The later monistic doctrines, designed to overcome the Cartesian dualism - e.g. by trying to reduce mental phenomena to mere products of the brain - did not succeed to solve the problem of free will in physics, namely that the freedom of action is a constitutive presupposition of all experimental science.

Since the Cartesian dualism is still the basis of the contemporary physical and engineering sciences, I will adopt it in spite of its incoherence. I am only too well aware of its limitations, nevertheless it is a temporarily useful fiction which ensures that matter does not contain spiritual elements in an essential way ${ }^{1}$. The reality here described must therefore be understood to be only that of a hypothetical purely material world. Moreover, I deliberately avoid any discussion of two deep unsolved problems of science - the issue of self-reference and the "phenomenon of nowness" [36]. Starting from this old-fashioned but not easily avoidable platform, it turns out that it is natural to associate endophysical theories of matter with ontically interpreted fundamental theories of matter which may be related to true natural laws. Here, the intended meaning of «truth» is related to some kind of objective independent reality, as opposed to a context-dependent intersubjective agreement.

Presumably, the endo/exo-relations discussed in this paper are more generally valid but I have to stress that I discuss here exclusively the endo/exo-dichotomy in the material domain. That is, I choose a very particular context, and if I use the term «contextindependent» I only mean independent of an additional context.

1 For a discussion of some possibilities to modify this requirement, compare [32]. 


\section{Rössler's endo/exo-distinction vs. quantum endo/exo-physics}

In order to avoid misunderstandings, I have to stress that Rössler's point of departure is quite different from mine. I accept the holistic nature of quantum physics as an irreducible trait of nature, while Rössler prefers to enlarge what I call "quantum endophysics" by a "more explicit" classical description in order to find some kind of an Archimedean point outside the empirical world from which one could explain what "really" happens in the socalled quantum world. For this, Rössler introduces a hypothetical external observer (a kind of superobserver with perfect observing power, like Laplace's or Maxwell's external demons) for whom quantum endophysics is supposed to be accessible from the outside [35]. From this perspective, there may be explicit external features which are known to the demon but which are not accessible from within ordinary quantum physics. On the other hand, there are quantum endophysically valid features which are absent in Rössler's explicit description [34, 35, 36]. For Rössler the ultimate reality is exophysical (accessible to the superobserver) while in my view the ultimate reality is endophysical, described by quantum endophysics which is at the most comprehensible by introspection. In spite of these very different metaphysical presuppositions, there are no logical contradictions - there is a peaceful coexistence between these two views.

Rössler adopted the paradigms of classical physics and computer science, and postulated the existence of an internal observer, that is an observer which can use only procedures and devices which are realizable from within the endo-system of which he is part. Rössler compared classical physics "from without" and classical physics "from within" and found that the associated state concepts were very different. He characterized classical endophysics with its internal observer as the study of demons which are blocked by a censor [34]. The aim of such studies is to understand the endoworld as seen by the internal observer in terms of an exophysical, uncensored superobserver.

In contrast, I choose quantum mechanics as theoretical starting point for the study of material reality. According to quantum mechanics, the material endoworld is holistic and not made out of any parts, so that the concept of an "internal observer" makes no sense in quantum endophysics. In this framework, it would be logically inconsistent to adopt the view that quantum endophysics deals with problems which arise when "the observers and their devices are inside the system they observe or act upon". In a consistent fully quantumtheoretical discussion we cannot neglect the existence of Einstein-Podolsky-Rosen correlations between any two material parts of the world. We can speak of an individual subject and an individual object only if these two systems are disentangled, that is, if we assume or if we can prove that they are not Einstein-Podolsky-Rosen-correlated. In this sense, quantum endophysics is conceptually different from classical endophysics, so that an elucidation of quantum endophysics proceeds in a quite different spirit. We may call the observer-free quantum endophysics the study of the Platonic heaven, the realm of nonspatial, non-mental, timeless but nevertheless real entities.

A hard-boiled positivist may have difficulties to appreciate such a quantum endophysics since it refers by definition to some kind of a Platonic universe, and not to empirical facts. The aim of the study of quantum endophysics is not the hope to find the "true and real" 
values for all endophysical observables ${ }^{1}$ (these do not exist!) but to formulate universally valid natural laws endophysically in view of theoretical derivations of operationally meaningful exophysical descriptions. As in the Boscovich-Rössler universe, in quantum theory a human observer can never observe the world as it is governed by the universal natural laws. Every operationally meaningful description we can give is observer-dependent or contextual. That is, for a hard-boiled positivist, universally valid natural laws do not exist. The introduction of a Platonic quantum endophysics is motivated by the necessity to find a place for universal natural laws.

\section{Why the standard reduction model does not work}

We speak of an epistemological reduction when one scientific theory is shown to be a deductive consequence of another one. The replacement of one theory by another may imply a methodological reduction but this is not the central issue. The main problem of reductionism (say in the sense of Kemeny and Oppenheim, or Ernest Nagel) is the question whether some or all phenomenological scientific theories can be deduced from a fundamental physical theory. While philosophers of science admit that "in practice it seems that very few examples of reduction exhibit a straightforward deductive pattern" [1], they fail to recognize that there is no single nontrivial example of a rigorous deduction of a phenomenological law from truly first principles ${ }^{2}$. The reason is that such a deduction is logically impossible without the introduction of a context. Scientists have studied many intertheoretical relations in a mathematically rigorous way and found conclusively that none of these correspond to any of the reduction relations defended by the philosophers. Phenomenological theories may be in a state of peaceful co-existence with first principles considered as fundamental, but they cannot be deduced from them without introducing crucial new structural elements.

Basic theories claim universal validity, while all phenomenological theories are contextdependent, they cannot be deduced from universally valid first principles without taking into consideration the abstractions evoked by the pattern recognition devices necessary for the observation of phenomena. Such context-dependent abstractions do not falsify our description of the material reality but they create the exophysical patterns by means of which we interpret the world. Of course, these exophysical patterns depend both on the intrinsic properties of the endoworld and on the abstractions associated with the pattern recognition procedure.

A mathematically formulated deduction of a higher-level description from a fundamental theory is conceivable only if in the basic theory a new contextual topology is introduced. This new topology is never given a priori but depends in a crucial way on the abstractions

1 Warning: Quantum mechanics is muddled with virtually the worst possible terminology. In the jargon of modern quantum mechanics, «observable» is a (most unfortunate) technical term with no fixed a priori meaning. In particular, the term «endophysical observable» has absolutely nothing to do with the exophysical concept of an "observation". In quantum endophysics, observables represent potential properties. In quantum exophysics statistical measurements are related with positive operator-valued measures (and not with observables).

2 The standard of rigor of the discussion about reductionism adopted by most philosophers of science is unacceptable. The level we have to require for any serious study of theory reduction has been set by Friedrichs [14] in his analysis of the relation between geometrical optics and electrodynamics. 
made by the cognitive apparatus or the pattern recognition devices used by the experimentalist. The closure of such a higher-level description in the contextual topology generates new context-dependent entities which are not already present in the fundamental description. The associated breakings of fundamental symmetries may be describable as singular asymptotic expansions which are not uniformly convergent in the intrinsic topology but only in an appropriate, non-fundamental, contextually selected topology. In this mathematically precise sense, one can speak of the emergence of novelty in descriptions on a higher level. The task of higher-level description is not to approximate the fundamental theory but to represent this contextual novelty. Yet, the universally valid first principles are never sufficient for deducing higher-level theories since, of course, the context is never given by first principles. The laws of nature do not determine uniquely the world we encounter, the specification of the context is at least of equal importance as the first principles.

Early modern science owes its success in part to an adherence to atomism, reductionism and a naive common-sense realism, i.e. the belief that ultimately everything comes down to the mechanics of very concrete atoms ${ }^{1}$. It started with the trustful presupposition that we can know things as they really are through observations and experiments, and naively related first principles with directly observable phenomena. In the course of time, however, theoretical physics developed more and more towards universal, context-independent theoretical formulations. This development cannot be explained positivistically from the mere desideratum to achieve intersubjective agreement about empirical facts. While Newton did not doubt the concrete existence of corpuscular entities in analogy with gross bodies ${ }^{2}$, the research program of atomism finally led to the formulation of quantum mechanics in terms of abstract elementary entities which are nothing like Newton's corpuscles but bear a striking resemblance to primordial archetypal structures. Nowadays, a rash identification of the concrete with primordial symbolic structures is untenable.

Modern theories of matter are most certainly not what famous philosophers of science like Joseph Sneed claim, namely that "scientific theories are sets of statements; some of which are empirically true or false" [37]. In modern physics is that what one may consider as "ultimate reality" quite universal but very abstract, and by no means directly related to the richness of the concrete. Claiming real existence for abstract ultimate entities necessarily has a Platonic flavor and is, of its nature, not provable. On the other hand, all so-called "facts" and empirical truths are heavily context-dependent, hence not in a straightforward manner related to universal physical laws which rule modern fundamental theories.

Faced with this situation, we are forced to distinguish between intrinsic and operational descriptions. An intrinsic description characterizes the properties and the states of a physical system without explicit reference to other physical systems. By contrast, an operational description makes explicit reference to other physical systems than the one singled out for

1 As late as 1872 Emil du Bois-Reymond said: "Denken wir uns alle Veränderungen in der Körperwelt in Bewegungen von Atomen aufgelöst, die durch deren konstante Zentralkräfte bewirkt werden, so wäre das Weltall naturwissenschaftlich erkannt." [4].

2 Recall Newton's ideas about atoms: "God in the Beginning form'd Matter in solid, massy, hard, impenetrable moveable Particles ...; no ordinary Power being able to divide what God Himself made one in the first Creation" ([22], Query 31). 
special study. It typically characterizes the properties of the object system in terms of test procedures which must be described in terms of external systems. The interrelations between intrinsic and operational descriptions are far more complex than presumed by the reductionists. For a deeper study, we are forced to introduce a crucial distinction, that between endo- and exo-theories. The research program of the reductionist has to be replaced by a study of the interface between endo- and exophysics.

Endophysics - and not exophysics - is the domain where one can hope to find universally valid first principles. My main thesis is that the endo/exo perspective is the proper tool to discuss both intrinsic and extrinsic descriptions in a common unifying framework, and to avoid the widespread confusion between ontic and epistemic notions. The idea that natural laws may be associated to ontological truths is nowadays not a popular view among scientists but it can hardly be avoided if one would like to keep realism and avoid naive realism. I will illustrate this thesis by the modern quantum theory of matter.

\section{On the ignorance of the endo/exo-dichotomy}

One may argue that the endo/exo distinction is unimportant since, after all, classical physics has been interpreted without too much difficulties without such a distinction. Historically, the measurement problem in classical physics has played no important role. Nevertheless, a thoughtful discussion of the relationship of first principles and phenomena shows that even in classical physics one cannot avoid to investigate the interface between endo- and exodescriptions.

The crucial point is that the referents and the aims of endophysics and of exophysics are different. Endophysics refers to a subject-independent reality while exophysics refers to the empirical reality. Endophysics aims at metaphysical universal laws, while exophysics aims to give us empirically adequate descriptions. Endo-entities belong to the subjectindependent reality, they may be related to hypothetical "things-in-themselves" or to Platonian ideas. They are hidden from us and certainly not directly observable. Observations and experiments do not reveal us directly this endo-reality. In contradistinction, exophysics refers also to tangible objects we can directly see, feel, and touch. The relations between independent reality and appearance, that is, the interface between endo- and exotheoretical descriptions is, strange to say, one of the most neglected topics of scientific discourse.

One reason for this disregard is the confusion of abstraction and approximation. Classical physics deals with «ideal entities» like mass points. Mass points do not exist in the real world, but in celestial mechanics they are considered as approximations, say describing the motion of real planets. On the other hand, Newtonian mass points have also an exact meaning als elementary systems in the sense of indecomposable representations of the Galilei group. Elementary systems are abstractions motivated by experiments but it would be a category mistake to identify the ideal object «mass point» (in the sense of an elementary system) with concrete mass particles (in the sense of an approximation). Ideal objects (like elementary systems, which may even lack individuality) belong to the endoworld while tangible things are the objects of an exophysical description. 
If one disregards the essential difference between the endo- and the exo-theoretical view, one can hardly avoid Bas van Fraassen's [13] conclusion that we should discard the idea that there are "laws of nature", or Nancy Cartwright's [5] verdict that the fundamental laws of physics "do not get the facts right", "do not state the truth" but that "phenomenological laws are indeed true of the objects in reality". These consequences are indeed inescapable if one assumes that the referents of fundamental theories are the phenomena in the exo-domain. However, such a view is based on a philosophical conception of the role and the aim of theoretical physics which few creative theoreticians will stand up for. Fundamental scientific theories do not move onward solely by generalizations of phenomenological laws and the rational use of rational principles. In the wording of Marcus Fierz: "Theoretical physics surely appears quite rational, but it rises from irrational depths." [9]. The structure of endophysics cannot be dealt with without considering the symbolic construction of scientific theories ${ }^{1}$.

Theoretical scientists have a deep-rooted bias favoring symmetry. Lie groups and gauge invariance are now recognized as basic principles, but there is no logical way from experience to such symbolic forms. Endophysical first principles depend to an astonishing degree on mathematical constructs like symmetries which in turn depend on primordial intuition $^{2}$ and symbolic representations ${ }^{3}$. It is impossible to comprehend the structure of endophysics if one dismisses the inherent human capacity for concept formation. It is a necessary element in the creation of fundamental theories so that these always contain archetypal elements which do not come in directly via physical experimentation, and which reason fails to grasp. The process of forming physical theories has been described by Wolfgang Pauli as follows: "Ich hoffe, daß niemand mehr der Meinung ist, daß Theorien durch zwingende logische Schlüsse aus Protokollbüchern abgeleitet werden, eine Ansicht, die in meinen Studententagen noch sehr in Mode war. Theorien kommen zustande durch ein vom empirischen Material inspiriertes Verstehen, welches am besten im Anschluß an Plato als zur Deckung kommen von inneren Bildern mit äußeren Objekten und ihrem Verhalten zu deuten ist. Die Möglichkeit des Verstehens zeigt aufs neue das Vorhandensein regulierender typischer Anordnungen, denen sowohl das Innen wie das Außen des Menschen unterworfen sind." [25].

Cartwright correctly states that "phenomenological laws describe what happens", but that the fundamental laws do not state the facts. This is trivially true since the referent of endophysical first principles is not the exoworld of empirical facts. Endo-concepts are

1 In direct contrast to a sign, a symbol never stands for a known thing. We use the concept of a symbol in the sense of Carl Gustav Jung, as "an expression for something that cannot be characterized in any other or better way". ([18], \#816). "Since every scientific theory contains a hypothesis, and is therefore an anticipatory description of something still essentially unknown, it is a symbol." ([18], \#817). Compare also the the essays by Hermann Weyl [42, 43, 44].

2 Here, intuition is understood in the sense of a perception of primordial images via the unconscious. In spite of Brouwer's aversion from science, his "Primordial Intuition" is related to the intuition which is at work in theoretical science. For a discussion of Brouwer's Primordial Intuition, compare [38].

3 In a letter to Hermann Levin Goldschmidt Wolfgang Pauli writes: „Das Symbol ist stets ein abstraktes Zeichen, sei es nun quantitative oder qualitative, sei es mathematisch-gedanklich oder emotional bewertet (,gefühls-geladen“). Nur ein Teil des Symbols ist durch bewusste Ideen ausdrückbar, ein anderer Teil wirkt auf den „unbewussten“ oder „,vorbewussten“ Zustand des Menschen. So geht es auch mit den mathematischen Zeichen, denn nur derjenige ist für Mathematik begabt, für den diese Zeichen (im erläuterten Sinne) Symbolkraft besitzen. Das Symbol ist stets ein Gegensätze vereinigendes tertium, das die Logik allein allerdings nicht „geben“" kann.“[23]. 
linked to the universality and simplicity of primordial patterns, while exo-concepts relate to the variety and richness of the concrete and particular. Accordingly, endo-concepts are categorically different from exo-concepts. The crux in this discourse is that in the historical development of physics endo- and exo-descriptions have not been separated in a proper way.

The space-time concepts are particularly difficult since they play an important - but essentially different - role in endo- and in exophysics. Because of this double role we have to be careful not to confuse them. In contrast to the reversible Newtonian time which manifests no asymmetry between past and future, Norbert Wiener [45] spoke of Bergsonian time when he wanted to emphasize the novelty-creating aspect of time. Newtonian time refers to endo-theories, Bergsonian time to exo-theories. Freedom of action, according to Henri Bergson, is fully certified by direct experience, hence something associated to Bergsonian (in contrast to Newtonian) time [2]. In this sense, the freedom of the experimenter to choose initial conditions is an exophysical quality which is not necessarily in contradiction with a presupposed endophysical determinism.

\section{Ontic and epistemic interpretations}

An interpretation refers to a logically consistent theoretical formalism which is in a mathematically rigorous way codified. I adopt the following characterization:

\section{Definition: An interpretation of a mathematically formulated physical theory is characterized by a set of normative regulative principles which can neither be deduced nor be refused on the basis of the mathematical codification.}

We distinguish between epistemic and ontic interpretations. Epistemic interpretations refer to our knowledge of the properties or modes of reactions of systems, while ontic interpretations refer to the properties of the object system itself, regardless of whether we know them or not, and independently of any perturbations by observing acts. The operationalistic view requires an epistemic interpretation and usually works with a statistical ensemble description.

Both in endophysics and in exophysics, I will adopt a realistic view. In endophysics, the basic tenet requires that there is something that does not derive its existence from the existence of human mind. This "something" has been called independent reality by Bernard d'Espagnat [7]. Quite differently, the exophysical reality refers to observable phenomena, and corresponds to d'Espagnat's empirical reality. Statements about the empirical reality contain a non-removable reference to the community of possible observers. Such statements are at the most intersubjectively valid, or, in d'Espagnat's terminology, weakly objective. In contradistinction, hypothetical statements about the endophysical reality are strongly objective, they refer to the properties the endophysical reality supposedly has. Folklore to the contrary notwithstanding, quantum laws are strongly objective provided they are formulated in the framework of quantum endophysics. 
From an endophysical viewpoint, the Platonic ideas are standards of truth. That is, we hold that the assertions of an endophysical theory are true or false, and what make them true or false is not our mind or our experiments. Of course, we do not know, but we may hope that quantum endophysics gives a true description of the basic structure of the material world. In order that such an endotheory can be helpful for experimental science, it has to be supplemented by a relevant exophysical context which at best is appropriate. Certainly contextual exophysical propositions do not deserve the attribute «true». In modern experimental science, the generally accepted exophysical correctness criterion is Vico's verum factum principle: we can know for certain only that which we ourselves have made ${ }^{1}$.

A SHORT CHARACTERIZATION OF ENDO- AND EXO-DESCRIPTIONS

\begin{tabular}{|c|c|c|}
\hline & ENDO-DESCRIPTION & EXO-DESCRIPTION \\
\hline Regulative principle & $\begin{array}{c}\text { Universality: } \\
\text { Platonian simplicity } \\
\text { of the abstract and general }\end{array}$ & $\begin{array}{l}\text { Contextuality: } \\
\text { Aristotelian richness and variety } \\
\text { of the concrete and particular }\end{array}$ \\
\hline Laws & Fundamental first principles & Phenomenological \\
\hline Referent of the theories & $\begin{array}{c}\text { Hypothetical } \\
\text { "things-in-themselves", } \\
\text { Platonian ideas }\end{array}$ & $\begin{array}{l}\text { Concrete objects } \\
\text { directly perceptible phenomena, } \\
\text { empirically accessible phenomena }\end{array}$ \\
\hline Truth criterion & Platonic & Verum factum principle \\
\hline Natural interpretation & Ontic & Epistemic \\
\hline Logic & $\begin{array}{c}\text { Sharp logic } \\
\text { (Boolean or orthomodular) }\end{array}$ & $\begin{array}{c}\text { Fuzzy logic } \\
\text { (Boolean or orthomodular) }\end{array}$ \\
\hline Descriptions & $\begin{array}{l}\text { Descriptions are within } \\
\text { certain limits universal, } \\
\text { but not operational }\end{array}$ & $\begin{array}{l}\text { Descriptions are operational } \\
\text { but in a crucial sense } \\
\text { context-dependent }\end{array}$ \\
\hline Parameters & $\begin{array}{c}\text { Very few } \\
\text { universal constants }\end{array}$ & $\begin{array}{l}\text { Many context-dependent } \\
\text { empirical constants }\end{array}$ \\
\hline Time & $\begin{array}{l}\text { Newtonian time, } \\
\text { no asymmetry between } \\
\text { past and future }\end{array}$ & $\begin{array}{l}\text { "Bergsonian" time, } \\
\text { asymmetry between } \\
\text { past and future }\end{array}$ \\
\hline Events & No facts & Emergence of facts \\
\hline
\end{tabular}

1 "Verum et factum convertuntur" ("the true and the made are convertible"), Giovanni Battista Vico (16681744) in: De antiquissima Italorum sapientia, 1710. Compare also [41], Ziff. 331, and sects. 1.2 and 2.3 of the introduction by $\mathrm{V}$. Hösle. 


\section{On the relations between quantum endophysics and quantum exophysics}

Science has always been looking for unity in diversity. Unifying endotheoretical first principles have to be context-independent. The Aristotelian richness and variety of the concrete and particular has no place in endophysics. At all times, the guideline for the formulation of first principles has been simplex sigillum veri - radical structural simplicity in the sense of simple primordial ideas. An exemplary primordial idea is atomism. Democritos suggested that in the attempt to divide matter again and again, one finally should end up with indivisible, unchangeable entities, called atoms. The mechanistic program of atomism aimed at the explanation of all qualities of matter in terms of the positions and motions of these atoms. In the seventeenth century atomism has already been established as a viable natural philosophy and was one of the driving forces of the development of physics. Ironically, according to our present understanding, we have to admit that all atomic hypotheses were wrong. The fundamental structures turned out to be much more abstract than the atomists hoped for. In fundamental quantum mechanics, context-independent material atoms or "elementary particles" do not exist. The modern view is much nearer to Plato's ideas, according to which the attempt to divide matter again and again results in mathematical forms. Theoretical physics progressed from the simple to the yet simpler, ending with the pure simplicity of fundamental symmetries. The associated elementary entities are defined as indecomposable representations of the symmetry group, yet matter is not made out of elementary entities (like bare electrons or bare fields).

All fundamental universally valid first principles we know are deterministic and refer to strictly closed systems ${ }^{1}$. Since strictly closed systems cannot be observed from outside, universal first principles are endophysical laws. Strictly speaking, there is nothing outside a quantum endosystem. The endophysical description is a view without perspective, it is God's panorama, a "view from nowhere". Endophysical first principles are inspired by the shadows on the wall of Plato's cave, but they cannot be deduced from experimental facts. The leading idea is simply that a fundamental law must not depend on contexts: it refers to an endoworld having a maximal unbroken symmetry. The associated endophysical elementary systems are precisely defined, and they are simple in the sense that they have unlike Newtonian atoms - only an exhaustible set of attributes. However, endophysical elementary quantum systems are not localized and lack individuality. Since in endophysics, the concept of an environment makes no sense, endophysical elementary systems have to be considerd as "naked" - no environmental effects are included.

In contradistinction to quantum endophysics, quantum exophysics is associated with experimental physics. Every experiment and every operationally meaningful description requires a division of the endo-world into an object system and an observing system. Yet, the endoworld does not present itself already divided - we have to divide it. Therefore endophysical first principles are not sufficient for an operational description of an exosystem, we have to add the particular context which characterizes the cut between the material object and the material observing tools.

1 A physical system is said to be closed if all the variables that can influence the behavior of the system have been taken into account in the initial specification. Any other system is called open. 
In quantum theories, the concepts of «object» and «observing tools» are conceptually highly nontrivial since a material system qualifies as an observing tool only if it is not entangled with the object by Einstein-Podolsky-Rosen correlations. An exophysical object is characterized by a cut which separates it from its environment, it owes its existence to the interaction with the environment. In particular, the separability and localizability of the exoobjects of our everyday experience are generated by interactions of the separated object with the environment. The cut between object and environment can not be chosen arbitrarily, nevertheless it is not prescribed by endophysical first principles. Exophysical objects are clearly abstraction-dependent, hence contextual, but they are not free inventions. They have no endophysical existence, but they represent patterns of the endo-reality. Exophysical objects are to some degree fuzzy, and carry with them a variety of connotations, so a precise description in terms of a sharp logic is not feasible - they are far too vague for that. The proper mathematical tool for their description is a fuzzy logic, in quantum exophysics realized by probability operator measures (i.e. positive operator-valued measures).

Another crucial difference between quantum endophysics and quantum exophysics is that there are no universally valid principles for exotheoretical descriptions of matter, but only contextual phenomenological laws. It is typical for such laws (like the equation for heat conduction, thermodynamical laws, information theory) that they contain new concepts (like temperature, entropy, information, memory, purpose, meaning), and many empirical parameters (like diffusion coefficients, Reynolds numbers, virial coefficients), which are unknown in fundamental endophysical theories.

An endophysical universe is Platonic, it has no manifest appearance, it cannot be observed directly by our five senses. In contrast to this unbroken wholeness, the world we experience is full of broken symmetries. The fundamental symmetries of the endo-world are not manifest in our everyday experience. It is necessary to break fundamental symmetries, as clearly recognized by Pierre Curie [6]: "C'est la dissymétrie qui crée le phénomène". Observable patterns of the world do not exist in the Platonic universe, they come into being through symmetry breakings. All symmetry breakings are contextual, all apparent violations of basic natural laws come from contextual symmetry breakings.

The relation between quantum endophysics and quantum exophysics corresponds to that of Plato's transcendent universals and the shadows on the wall of Plato's cave. The existence of such universals can only be inferred intuitively and expressed mathematically. The problem of linking endophysical ideas to exophysical empirical concepts is the task of a theory of measurement. The notorious measurement problem in quantum mechanics is an example of such an interface problem: the derivation of a statistical interpretation of quantum exophysics from ontologically phrased quantum endophysics ${ }^{1}$.

1 Most certainly, the historically so important but nevertheless ill-posed so-called "correspondence principle" will be of no use for this task. 


\section{Galilei-relativistic quantum mechanics}

For the further discussion, it is neither expedient nor feasible to start with the most fundamental first principles of physics. Yet even so, in the Galilei-relativistic domain, quantum mechanics is a highly successful theory of matter. In its most basic formulation, it fulfills most criteria of an endophysical theory. It refers to Platonic elementary systems (like bare electrons and photons) which can be characterized by an exhaustible set of attributes but which are not directly observable ${ }^{1}$. The dynamics of these quantities is formulated in terms of Newtonian time, which does not distinguish between past and future. Moreover, these endophysical dynamical laws are Hamiltonian, that is they are valid only for strictly closed systems. If we adopt the Cartesian cut, all first principles of this quantum endomechanics are strictly subject-independent, they refer to hypothetical "things-inthemselves". Therefore, in an appropriate codification ${ }^{2}$, Galilei-relativistic quantum mechanics may serve as a mathematical model for an endophysical theory of matter.

It is astonishing but correct that these metaphysical mathematical constructs are of prime importance for experimental science and engineering. Admittedly, the corresponding endo/exo-interface - the quantum mechanical measurement process - is the weakest feature of quantum mechanics. Nevertheless, we have pragmatic working rules (like the so-called projection postulate) which fix the gap for the time being. The only reasonable explanation why the first principles of quantum mechanics are so enormously successful in engineering science is that the endophysical laws are to some degree a true description of objective structural aspects of the material reality. If this is so, they " $d o$ state the truth".

Our contemporary texts on quantum mechanics fail to analyze the exo/endo-perspective and they mix up endo- and exo-theoretical notions. One of the reasons of this misconception is due to the fact that in traditional quantum mechanics - in contrast to classical mechanics the ontic endophysical and the epistemic exophysical description can be formulated in the very same mathematical framework: the Hilbert-space formalism of quantum mechanics. If the underlying phase space is locally compact, this procedure is mathematically correct but conceptually misleading. A closer analysis shows that for Hamiltonian theories (including classical mechanics and quantum mechanics over an arbitrary phase space) in general the mathematical formalism for ontic endophysics is different from the formalism for statistical exophysics.

Quantum endophysics describes material reality in terms of a non-fuzzy orthomodular, nonBoolean logical structure. Every exophysical description of this non-Boolean quantum reality involves a projection onto a Boolean context. Non-Boolean scientific theories (like quantum mechanics) intrinsically lead to the logical concept of complementarity. Contextindependent Boolean descriptions of a non-Boolean world do not exist.

1 For example, in the framework of Galilean quantum endophysics, a bare electron is uniquely characterized by its mass, spin, electric charge and magnetic dipole moment. Such a bare electron, however, is not observable because it is necessarily coupled with its own electromagnetic radiation field which does not belong to the attributes of the bare electron. For instance, the experimentally measurable mass of the (dressed) electron is not the mass of the bare electron.

2 Of course, pragmatic working rules like the projection postulate, or phenomenological concepts like entropy are not part of endophysics. 
Example: Hilbert-space endophysics is different from Hilbert-space exophysics

Often it is not appreciated that even the mathematical formalism for traditional statistical quantum exophysics is different from the formalism for individual quantum endophysics. Traditional quantum mechanics deals with locally compact phase spaces (i.e. systems with only finitely many degrees of freedom), so that in this special case the uniqueness theorem by Stone and von Neumann $[20,39]$ implies that both quantum endophysics and quantum exophysics can be formulated on the very same Hilbert space $\mathscr{H}$ as the basic mathematical substrate. This is a very unusual situation which easily leads to conceptual confusions.

In the traditional exophysical formulation, the algebra of bounded contextual observables is the $\mathrm{W}^{*}$-algebra $\mathscr{B}(\mathscr{H})$ of all bounded operators acting on the Hilbert space $\mathscr{H}$, while the statistical states are described by density operators, i.e. by elements of the Banach space $\mathscr{B}^{1}(\mathscr{H})$ of trace-class operators on $\mathscr{H}$, whereby $\mathscr{B}^{1}(\mathscr{H})$ is the predual of $\mathscr{B}(\mathscr{C}),\left\{\mathscr{B}^{1}(\mathscr{H})\right\}^{*}=\mathscr{B}(\mathscr{C})$. In the endophysical formulation, the algebra of intrinsic observables is much smaller, it is given by the $\mathrm{C}^{*}$-algebra $\mathscr{B}^{\infty}(\mathscr{H})$ of compact operators acting on $\mathscr{H}$, while the individual endophysical states can be represented by the positive extremal elements of the dual $\left\{\mathscr{B}^{\infty}(\mathscr{H})\right\}^{*}$ of the $\mathrm{C}^{*}$-algebra $\mathscr{B}^{\infty}(\mathscr{H})$. It turns out that $\left\{\mathscr{B}^{\infty}(\mathscr{C})\right\}^{*}=\mathscr{B}^{1}(\mathscr{H})$. This is a singular situation, implying that the state space of individual quantum mechanics is the same Banach space as the state space of statistical quantum mechanics, a fact which is responsible for many confusions in the literature. Many of the conceptual difficulties and alleged paradoxes of traditional quantum mechanics are due to the failure to distinguish properly between endophysical and exophysical formulations.

In traditional quantum mechanics, the $\mathrm{C}^{*}$-algebra $\mathscr{B}^{\infty}(\mathscr{H})$ of intrinsic observables determines uniquely the $\mathrm{W}^{*}$-algebra $\mathscr{B}(\mathscr{H})$ of the statistical description, $\left\{\mathscr{B}^{\infty}(\mathscr{C})\right\}^{* *}=\mathscr{B}(\mathscr{C})$. This mathematical fact - essentially the Stone-von Neumann uniqueness theorem - suggests that traditional quantum mechanics is not a suitable candidate for the description of the universal endophysical first principles. In this framework, there would be no symmetry breakings, and all the richness and variety of the exophysical empirical reality could not exist.

\section{Ontic quantum endophysics and epistemic quantum exophysics}

The appropriate interpretation of endophysics is an individual interpretation, preferably an ontic one. Since there is no concept of an observer, an epistemic interpretation makes no sense. In the algebraic codification of physical theories, an individual interpretation requires the concept of an individual state which in turn requires as a mathematical substrate a continuous structure like a manifold or a separable $\mathrm{C}^{*}$-algebra. An individual state is called an ontic state if it realizes the intrinsic concept of Platonic truth. Note that an ontic state 
does not provide us with any operational criterion for determining it. Experimentally inaccessible ontic states are not meaningless but play a particularly interesting role in classical mechanics, they lead to the phenomenon of the so-called deterministic chaos, that is, dynamical processes whose endophysical description is bidirectionally deterministic but all whose exophysical operational descriptions are nondeterministic.

In order that exophysics has a direct relation to experiments, its interpretation has to be operational and to refer to our knowledge. In the algebraic codification of physical theories, an epistemic interpretation requires the concept of probability measure, or more generally, of statistical states. The appropriate mathematical structures are measurable spaces, like Lebesgue spaces, or $\mathrm{W}^{*}$-algebras with a separable predual.

\section{Example: Ontic states in classical mechanics of individual mass points}

Consider a classical dynamical system with a symplectic manifold $\Omega$ as phase space. For every point $\omega \in \Omega$, the equations of motion of the dynamical system specify a trajectory $t \mapsto \omega(t), t \geq 0$. The quantity $\omega(t)$ is called the individual state at time $t$. This individual state $\omega(t)$ describes in an exhaustive manner all the properties the individual dynamical system has at time $t$, and can therefore be interpreted as an ontic state, referring to intrinsic properties an object has at time $t$.

\section{Example: Epistemic statistical states in classical mechanics}

Consider a classical dynamical system with phase space $\Omega$. For a statistical description one introduces a measurable space $(\Omega, \mathcal{B})$, where $\mathcal{B}$ is the most refined Boolean algebra of experimentally decideable events. A countably additive probability measure $\mu$ on the measurable space $(\Omega, \mathcal{B})$ is called a statistical state of the dynamical system with the phase space $\Omega$. The probability measure $\mu$ refers to our knowledge, usually to the results of statistical experiments. As a rule, we cannot know the individual state $\omega$ but only that it is more likely to be in some Borel sets of $\Omega$ than in others. That is, $\mu(B)$ is interpreted as the probability that the individual state $\omega$ is in the Borel set $B \in \mathcal{B}$. In the algebraic formulation, the statistical states are represented by probability densities, that is of positive and normalized elements of the Banach space $L^{1}(\Omega)$. The dual of this Banach space is the $\mathrm{W}^{*}$-algebra $L^{\infty}(\Omega)$ of bounded Borel-measurable functions on $\Omega$, and is called the algebra of bounded observables.

It is generally accepted that quantum mechanical predictions are intrinsically of statistical character. This circumstance, however, neither implies that an individual interpretation of quantum mechanics is impossible, nor that a purely statistical interpretation is unproblematic. In quantum theories, the statistical state space is not a simplex, so that a statistical quantum state does not specify an ensemble in the sense of a mixture of individual systems in pure states. Moreover, the statistical interpretation presupposes the existence of classical domains, the existence of irreversible events, and a preferred direction of time such that the concept of prediction makes sense. These are clearly contextual requirements which should 
not be included in the collection of first principles but they have to be used as regulative principles in a derivation of a statistical description from the basic individual interpretation.

Just as in classical point mechanics, the individual description of an arbitrary quantum system can be given in terms of an appropriate separable $\mathrm{C}^{*}$-algebra $\mathscr{}$ of intrinsic observables, where the individual states are represented by the extremal positive elements of the dual $\mathscr{C}^{*}$ of $\mathscr{C}$. The statistical description of a quantum system has to be given in terms of an appropriate $\mathrm{W}^{*}$-algebra $\mathscr{M}$ of contextual observables where it is assumed that $\mathscr{M}$ has a separable predual $\mathscr{M}_{*},\left(\mathscr{M}_{*}\right)^{*}=\mathscr{M}$. In quantum theories the algebras $\mathscr{N}$ and $\mathscr{M}$ are in general noncommutative. In the special case of commutative algebras we speak of classical quantum systems, and we can represent these algebras as in historical classical mechanics by $\mathscr{\mathscr { C }}=\mathscr{C}^{\infty}(\Omega)$ and $\mathscr{M}=L^{\infty}(\Omega)$, where $\mathscr{M}_{*}=L^{1}(\Omega)$.

\section{Example: Individual and ontic states in quantum endophysics}

The universe of discourse is characterized by a separable $\mathrm{C}^{*}$-algebra $\mathscr{C}$ of intrinsic observables. The selfadjoint elements of $\mathscr{C}$ represent the potential properties of the universe of discourse. The ontic state at time $t$ is mathematically represented by a normalized extremal positive linear functional $\rho_{t}$ on $\mathscr{\Upsilon}$. A potential property, represented by the selfadjoint element $A \in \mathscr{A}$, is said to be actualized at time $t$, if $A$ is dispersionfree with respect to the ontic state $\rho_{t}$, that is if $\rho_{t}\left(A^{2}\right)=\left\{\rho_{t}(A)\right\}^{2}$. In this case we attribute the value $\rho_{t}(A)$ to the observable $A$, and we say the observable $A$ has the value $\rho_{t}(A)$ at time $t$. If an observable is not dispersionfree, it is not actualized, and we attribute no value to it. That is, the potential properties characterize the universe of dicourse, while the actualized properties characterize the state of the universe of discourse ${ }^{1}$.

This ontic interpretation includes as a special case the traditional realistic interpretation of classical point mechanics over the phase space $\Omega$. In this case, the algebra of intrinsic observables is given by the $\mathrm{C}^{*}$-algebra $\mathscr{\alpha}=\mathscr{C}^{\infty}(\Omega)$ of continuous functions on $\Omega$ which vanish at infinity. The ontic states are represented by the points of $\Omega$, or, by Gelfand's theorem, equivalently by the extremal positive elements of the dual of $\mathscr{C}^{\infty}(\Omega)$. Since the $\mathrm{C}^{*}$-algebra $\mathscr{C}^{\infty}(\Omega)$ is commutative, all observables are actualized at every instant.

1 In the traditional Hilbert-space formalism with a separable Hilbert space $\mathscr{H}$, the algebra $\mathscr{\nearrow}$ of intrinsic observables is given by the separable $C^{*}$-algebra $\mathscr{\mathscr { C }}=\mathscr{B}^{\infty}(\mathscr{H})$ of compact operators acting on $\mathscr{H}$. There is a one-to-one correspondence of ontic states and rays in $\mathscr{H}$, so that every ontic state $\rho_{t}$ can be represented by a normalized state vector $\Psi(t) \in \mathscr{H}$. An observable $A$ is actualized if and only if the state vector $\Psi(t)$ is eigenvector of $A$; in this case $A$ has the value $\langle\Psi(t) \mid A \Psi(t)\rangle$. 


\section{Example: Statistical and epistemic states in Hilbert-space quantum mechanics}

The irreducible Hilbert-space representation of traditional quantum mechanics is an exophysical statistical description and starts with a separable Hilbert space $\mathscr{H}$. The associated algebra $\mathscr{B}(\mathscr{H})$ of all bounded linear operators acting on $\mathscr{H}$ plays the role of the algebra $\mathscr{M}$ of bounded contextual observables, $\mathscr{M}=\mathscr{B}(\mathscr{H})$. Traditional quantum mechanics uses the concept of statistical states which refer to the outcome of statistical experiments. They enjoy the property of $\sigma$-additivity and are described by normalized positive elements of the predual $\mathscr{B}(\mathscr{C})_{*}$ of the algebra $\mathscr{R}(\mathscr{H})$ of contextual observables, $\left\{\mathscr{R}(\mathscr{H})_{*}\right\}^{*}=\mathscr{P}(\mathscr{H})$. The predual $\mathscr{B}(\mathscr{C})_{*}$ is isomorphic to the Banach space $\mathscr{B}^{1}(\mathscr{H})$ of all trace-class operators, $\mathscr{B}(\mathscr{H})_{*} \cong \mathscr{B}^{1}(\mathscr{H})$. Therefore, a statistical state $\rho$ of traditional quantum mechanics can be represented by a positive trace-class operator $D_{\rho}$ of unit trace,

$$
\begin{gathered}
\rho \leftrightarrow D_{\rho}, \quad D_{\rho} \in \mathscr{B}^{1}(\mathscr{H}) \quad, \quad D_{\rho} \geq 0 \quad, \operatorname{tr}\left(D_{\rho}\right)=1, \\
\rho(A)=\operatorname{tr}\left(D_{\rho} A\right) \quad \text { for every } A \in \mathscr{B}(\mathscr{C}) .
\end{gathered}
$$

The operator $D_{\rho}$ representing the statistical state $\rho$ is called density operator. Pure statistical states are represented by idempotent density operators.

\section{The Heisenberg cut between object and environment}

In all experimental science we have to split the world into two parts, the subject and the object. There are many possibilities for such a cut. The Cartesian cut requires a separation of the whole reality into mind and body. The Heisenberg cut $^{1}$ presupposes the Cartesian cut, it divides in addition the purely material universe of discourse into a material object and material observing tools. In choosing the Heisenberg cut we have some but not absolute freedom since the cut must satisfy specific physical requirements. In the mathematical framework of quantum mechanics, the Heisenberg cut requires a contextual tensor-product decomposition of the whole material reality such that there are no Einstein-Podolsky-Rosen correlations between the observed object and the observing tools. This cut is inevitably contextual since in endophysics there is no God-given Heisenberg cut, not even an intrinsic tensor-product structure.

Practical quantum mechanics presupposes that it is possible to dissect nature in such a way that the observing tools can be described in terms of classical physics or engineering science. The possibility of such a Heisenberg cut hinges crucially on an appropriate choice of the tensor-product factorization of the universe of discourse. A sufficient condition is that the relevant degrees of freedom of the measuring instrument constitute a classical quantum system $^{2}$.

1 Note that Heisenberg does not speak of a cut between observed system and observer but of a "Schnitt zwischen dem zu beobachtenden System und den Messapparaten" [16], and of the "Schnitt zwischen der Beschreibung durch Wellenfunktionen und der klassisch-anschaulichen Beschreibung" [15].

2 A quantum system is said to be classical if its algebra of observables is commutative. Note that every classical quantum system depends on Planck's constant. If there are no Einstein-Podolsky-Rosen correlations between the systems separated by the Heisenberg cut, then the existence of contextual classical quantum systems has not to be postulated, but is a consequence of algebraic quantum mechanics. The sufficient condition follows from the following theorem: Let $\mathscr{N}$ and $\mathscr{B}$ be two $C^{*}$-algebras and $\mathscr{C}=\mathscr{\Upsilon} \otimes \mathscr{B}$ 
In endophysics, the space-time structure is introduced by the covariance of the theory under a kinematic group (like the Poincare or the Galilei group), while all interactions are due to gauge fields [46]. This complex of ideas leads to a description of the endoworld in terms of bare elementary entities and an artificial splitting of the Hamiltonian into a "free part", describing bare elementary systems and bare fields, and "interactions" between the free parts. This endophysical tensorization leads to strong interactions and intense EinsteinPodolsky-Rosen correlations between these bare elementary systems and is therefore irrelevant for a tensorization appropriate for a Heisenberg cut. Starting with endophysical first principles, we know virtually nothing about the existence, the uniqueness or equivocality of exophysical tensor-product decompositions appropriate for an operational description.

That is, endophysical first principles are insufficient for a theory of human knowledge. A theory which describes observable phenomena cannot keep the human means of data processing out of consideration. Heisenberg's cut is necessary for the description of the patterns of the exophysical reality, it determines what we consider as relevant and what was irrelevant. The need for contingent elements is the price we have to pay for the operationalization of the endophysical first principles. In quantum endophysics, the notions "patterns" and "phenomena" have no meaning. In order to get observable patterns, we have to break the holistic symmetry of the endoworld by dividing it into an object system and an observing system. The associated pattern recognition projects the holistic, non-Boolean endoworld into an exophysical Boolean registration system. This projection is neither arbitrary nor unique. It is not arbitrary since all possible patterns are preexistent in the endoworld. But these preexistent patterns become manifest only in the appropriate exophysical tensor product decomposition.

The relationship between the endophysical first principles and the directly observable patterns is, however, notoriously difficult. In the mathematically formulated theory, it amounts to find a dressing transformation which changes the endophysical tensorization in terms of bare elementary systems to an exophysical tensorization such that the dressed objects are not (or only weakly) Einstein-Podolsky-Rosen-correlated with the environment ${ }^{1}$. By environment we simply mean everything which does not count to the object system under investigation - the rest of the world. At first sight, the distinction between object and environment is quite blurred when one considers the object and its environment not as separate static entities but as dynamically interacting systems. Fortunately, in the quantum-mechanical description there is an important criterium: In order that the concepts «object» and «environment» make any sense at all, the corresponding physical subsystems must not be entangled. The object may be in arbitrarily strong interaction with its environment, but during the whole lifetime of the object there must be no (or negligibly small) Einstein-Podolsky-Rosen correlations between object and environment.

their minimal tensor product. Every pure state $\gamma$ on $\mathscr{C}$ is of the form $\gamma=\alpha \otimes \beta$ for some pure states $\alpha$ of $\mathcal{\checkmark}$ and $\beta$ of $\mathscr{B}$ if and only if either $\mathscr{\mathcal { Y }}$ or $\mathscr{B}$ is commutative ([40], theorem 4.14).

1 In general, we have to expect that the Heisenberg cut, hence the relevant exophysical tensorization is time-dependent. Unfortunately, a theory of the dynamics of a Heisenberg cut seems to be beyond our present proficiency. 
A SHORT CHARACTERIZATION OF QUANTUM ENDO- AND EXOPHYSICS

\begin{tabular}{|c|c|c|}
\hline & $\begin{array}{l}\text { ENDO-QUANTUM- } \\
\text { MECHANICS }\end{array}$ & $\begin{array}{c}\text { EXO-QUANTUM- } \\
\text { MECHANICS }\end{array}$ \\
\hline Basic mathematical structure & $\begin{array}{c}\text { Continuous } \\
\text { (classical point mechanics: } \\
\text { manifolds) }\end{array}$ & $\begin{array}{c}\text { Measureable } \\
\text { (classical statistical mechanics: } \\
\text { Lebesgue spaces) }\end{array}$ \\
\hline Algebra of observables & $\begin{array}{c}\text { Separable nuclear } \\
C^{*} \text {-algebra } \mathscr{} \\
\text { (classical: commutative, } \\
\text { quantal: non-commutative) }\end{array}$ & $\begin{array}{l}\text { W*-algebra } \mathscr{M} \\
\text { with separable predual } \mathscr{K}_{*} \\
\text { (classical: commutative, } \\
\text { quantal: non-commutative) }\end{array}$ \\
\hline Observables & $\begin{array}{l}\text { Intrinsic observables: } \\
\text { Selfadjoint operators } A, \\
\qquad A \in \mathscr{\checkmark}\end{array}$ & $\begin{array}{c}\text { Contextual observables: } \\
\text { Probability operator measures } F \text {, } \\
\qquad \mathscr{M} \ni F: \Sigma \rightarrow \mathscr{M}\end{array}$ \\
\hline States & $\begin{array}{l}\text { Individual ontic states: } \\
\text { Extremal elements in the dual } \\
\text { of the algebra }\end{array}$ & $\begin{array}{l}\text { Statistical epistemic states: } \\
\text { Normalized positive elements of } \\
\text { the predual } \mathscr{M}_{*} \text { of } \mathscr{M}\end{array}$ \\
\hline Interpretation & $\begin{array}{l}\text { Individual ontic interpretation: } \\
\text { An intrinsic observable } A \in \mathscr{V} \\
\text { represents potential properties. } \\
\text { An observable } A \text { is actualized if } A \\
\text { is dispersion-free with respect to } \\
\text { the ontic state } \rho_{t}, \rho_{t}\left(A^{2}\right)=\rho_{t}(A)^{2} \text {. } \\
\text { In this case, } A \text { has the value } \rho_{t}(A) \text {. }\end{array}$ & $\begin{array}{l}\text { Statistical epistemic interpretation: } \\
\text { A probability operator measure } \\
F: \Sigma \rightarrow \mathscr{M} \text { and the statistical state } \\
\rho \in \mathscr{M}_{*} \text { generate the probability } \\
\text { measure } \mu: \Sigma \rightarrow \mathbb{R}^{+} \text {for a } \\
\text { predictive measurement, } \\
\mu(B)=\rho\{F(B)\}, B \in \Sigma .\end{array}$ \\
\hline Tensor product structure & $\begin{array}{l}\text { Determined by an ergodic } \\
\text { representation of a fundamental } \\
\text { symmetry group, giving rise to } \\
\text { "bare" elementary systems. }\end{array}$ & $\begin{array}{l}\text { Contextually reconstructed by the } \\
\text { requirement that exophysical } \\
\text { objects have to be disentangled. } \\
\text { Examples are "dressed particles". }\end{array}$ \\
\hline Referents of the theory & $\begin{array}{l}\text { Elementary systems which have } \\
\text { an exhaustible set of attributes, } \\
\text { but which are entangled } \\
\text { with everything }\end{array}$ & $\begin{array}{l}\text { Contextual objects which have } \\
\text { an inexhaustible set of attributes, } \\
\text { but which have to be disentangled } \\
\text { from the environment }\end{array}$ \\
\hline Time & $\begin{array}{l}\text { Endo-time is represented by } \\
\text { a one-parameter group }\end{array}$ & $\begin{array}{l}\text { Exo-time is represented by } \\
\text { a one-parameter semigroup }\end{array}$ \\
\hline Time operator & $\begin{array}{l}\text { No time operator for the } \\
\text { Newtonian time does exist }\end{array}$ & $\begin{array}{l}\text { A time operator for the } \\
\text { "Bergsonian" time may exist } \\
\text { (K-flow-type processes) }\end{array}$ \\
\hline Dynamics & $\begin{array}{c}\text { Deterministic } \\
\text { given by a time-reflection } \\
\text { invariant Hamiltonian } \\
\text { one-parameter group }\end{array}$ & $\begin{array}{c}\text { Irreversible } \\
\text { given by a dynamical } \\
\text { one-parameter semigroup }\end{array}$ \\
\hline
\end{tabular}




\section{On the importance of the environment}

The environment of an object system acts as background which is indefensibly neglected in historical quantum mechanics. From our first principles we have absolutely no arguments which would justify the neglect of the environment. The omnipresent Einstein-PodolskyRosen correlations imply that electrons, atoms, or molecules of the empirical reality never exist in total isolation. That is, the environment of an exophysical quantum system can never be left out of consideration. The environment induces symmetry breakings and is responsible for the perceptible separability of the empirical reality and the localizability of contextual objects.

Symmetry breakings are possible in quantum mechanics, though not in the historical Hilbert-space codification of quantum mechanics by Johann von Neumann [21]. This mathematical formalism stands and falls with the uniqueness theorem by Stone and von Neumann for the canonical commutation relations [20,39]. This theorem says that for locally compact phase spaces all Hilbert-space representations of the canonical commutation relations are physically equivalent, hence the Hilbert-space formalism is justified. However, for systems with non-locally-compact phase spaces this uniqueness theorem fails and there are infinitely many physically inequivalent and physically relevant representations, so that in this situation the von Neumann codification becomes useless. Nowadays, we know that inequivalent representations of the canonical commutation relations are indispensable, not only for quantum field theory and quantum statistical thermodynamics, but also for engineering physics, chemistry and biophysics. It is a simple corollary of this uniqueness theorem that symmetry breakings and classical quantum systems are impossible in the unnecessarily restricted von Neumann codification.

That is, the traditional Hilbert-space formalism is not an adequate codification, neither for quantum endophysics nor for the description of the environment coming forth in quantum exophysics. Fortunately, these difficulties fade away in the modern algebraic formulation of quantum mechanics. Algebraic quantum mechanics is nothing else but a precise and complete codification of the heuristic ideas of quantum mechanics of the pioneer days, it is valid for systems with finitely or infinitely many degrees of freedom and is physically equivalent to von Neumann's codification in the case of purely quantal finite systems. Algebraic quantum mechanics allows in a natural way the description of superselection rules and of classical quantum systems.

An often heard, but fallacious objection against the relevance of algebraic quantum mechanics is the claim that "the world is a system having only finitely many degrees of freedom". Evidently, this is not a physical but a metaphysical statement, an idea that dates from the atomism of Democritos. The possibility to count degrees of freedom is the result of a quite arbitrary normal-mode analysis. Exophysically speaking, a non-arbitrary criterion is whether the phase space for the description of the environment is locally compact or not. Since every material object system is coupled to the gravitational and to the electromagnetic field these fields cannot be neglected in the description of the environment of a contextual object. In a Hamiltonian description, these fields require phase spaces which are not locally compact. 


\section{From quantum endophysics to quantum exophysics}

The connection between fundamental endophysical theories and exophysical descriptions is nontrivial. It is related to the old enigma of the relationship between the simple, eternal, unchanging, absolute universals of Plato's reality and the rich complexity of particular spatiotemporal material objects of our direct experience. As recent science has moved to more and more abstractions, its basis has become more and more endo-theoretical, and the connection with directly perceptible phenomena has become less and less evident. The predicates of the endotheory cannot anymore be considered as approximately satisfied by palpable exophysical objects. That is, the endophysical reality is quite distinct from the exophysical reality, so that the explanatory and predictive power of endotheories needs to be explained.

All concepts of empirical science refer to observations obtained by some pattern recognition methods which abstract from many features, and concentrate on those aspects which we consider as relevant. What is considered as relevant and what as irrelevant is never given endophysically. Provided we are able to describe precisely what we consider as relevant and what as irrelevant, what our deliberate lack of interest is, then we can add the very same abstractions to the endophysical first principles. Such metatheoretical regulative principles break fundamental symmetries of endophysics. However, there is no universal principle for breaking endophysical symmetries, there are myriads of possibilities. The richness and variety of the exophysical concrete and particular come into being by a contextual symmetry breaking.

Symmetry breakings - which are inevitably necessary in order to divide the world in a part "which sees" and a part "which is seen" - arise only if physically inequivalent exophysical representations of the underlying endophysical structure exist. In the framework of algebraic quantum mechanics, such a situation can arise only if the basic endophysical system is sufficiently rich, that is, if the $\mathrm{C}^{*}$-algebra $\mathscr{C}$ of the endophysical intrinsic observables is essentially larger than the $\mathrm{C}^{*}$-algebra of compact operators (e.g. an anti-liminary $\mathrm{C}^{*}$-algebra). A most interesting feature of algebraic quantum mechanics is that it provides the mathematical tools which allow to step out of the endoworld, namely the GelfandNaimark-Segal-construction, or GNS-construction for short. The GNS-construction allows the construction of a context-dependent Hilbert-space $\mathscr{C}$ and a faithful representation $\pi(\mathscr{C})$ acting on the Hilbert space $\mathscr{C}$. The weak closure of $\pi(\mathscr{C})$ in the Hilbert space $\mathscr{H}$ is a $\mathrm{W}^{*}$-algebra $\mathscr{M} \supset \pi(\mathscr{C})$ which represents the algebra of contextual observables of an exophysical description. From a conceptual point of view it is not surprising that such a construction of quantum exophysics from quantum endophysics is highly nonunique. With the only exception of von Neumann's codification of traditional quantum mechanics (where the endophysical $C^{*}$-algebra is the algebra of compact operators), there are infinitely many physically inequivalent $\mathrm{W}^{*}$-representations of the underlying endophysical $\mathrm{C}^{*}$-algebra of intrinsic observables. These inequivalent $\mathrm{W}^{*}$-representations correspond to different exophysical descriptions of one and the same endosystem.

Note that the exophysical $\mathrm{W}^{*}$-algebra $\mathscr{M}$ is strictly larger than the faithful representation $\pi(\mathscr{C})$ of the $\mathrm{C}^{*}$-algebra of endophysical observables. That is, all endophysical observables appear also in exophysics but in addition there are new observables, also classical 
observables which commute with all observables, and spontaneously broken symmetries. However, inequivalent representations lead to different new observables and to different symmetries. We call such contextual new observables emergent ${ }^{1}$ in the sense that they are generated by the endophysical algebra $\mathscr{C}$ of intrinsic observables together with a context which selects a particular representation. Clearly, emergent exophysical observables are not functions of the elements of $\pi(\mathscr{C})$.

Since there are uncountably infinitely many inequivalent representations of the endophysical $\mathrm{C}^{*}$-algebra, the selection of the relevant ones is not an easy task. Every representation, hence every exophysical description, is related to a particular abstraction and idealization. An exophysical description is possible only by a deliberate lack of interest, a decision of what we consider as relevant and what as irrelevant. Such a choice is not written down in the endophysical first principles of the basic theory. However, we can in addition to the intrinsic norm topology of the endophysical $\mathrm{C}^{*}$-algebra introduce a new contextual topology which describes our ideas of what is relevant and what is not.

In the framework of the GNS-construction, such a contextually selected topology can be introduced via a particular positive linear functional $\rho$ on a $\mathrm{C}^{*}$-algebra $\mathscr{}$ of intrinsic observables. The pair $(\rho, \mathscr{C})$ gives rise to a (within unitary equivalence) unique cyclic representation $\left(\pi_{\rho}, \mathscr{H}_{\rho}, \Xi_{\rho}\right)$, called the GNS-representation of $\mathscr{}$ induced by $\rho$. The corresponding exophysical $\mathrm{W}^{*}$-algebra $\mathscr{M}_{\rho}$ of contextual observables (with respect to the topology induced by $\rho$ ) is then given by the weak closure of $\pi_{\rho}(\mathscr{A})$ in the algebra $\mathscr{B}\left(\mathscr{H}_{\rho}\right)$ of all bounded linear operators acting on $\mathscr{H}_{\rho}$.

Our ability to describe the world cannot go farther than our ability to isolate exophysical objects which have individuality and properties. They may change their actualized properties but they keep their identity. We adopt the view that exophysical objects are carriers of patterns, they arise in interaction with the rest of the world, and are always contextual and inherently fuzzy. Nevertheless, they reflect structures of the world. Nature's pattern emerge and become intelligible only with the active participation of the human mind. Contextual objects are constructed, not «things in themselves», they are phenomenal entities posited by the theory.

In quantum exophysics, we define an object as an open quantum system, interacting with its environment, but which is not Einstein-Podolsky-Rosen-correlated with the environment. Quantum systems which are not objects are entangled with their environments, they have no individuality and allow only an incomplete description in terms of statistical states. From the mathematical formalism of algebraic quantum mechanics it follows that the observing system necessarily must be a classical quantum system (in the sense of Boolean logical structure). In retrospect, this situation justifies Bohr's requirement that "the functioning of the measuring instrument must be described within the framework of classical physical ideas" [3]. This requirement is also in agreement with the fact that, without exception, every experimental arrangement of present-day physical science, and all experimental results in physics, chemistry and molecular biology can be described in engineering terms, using only concepts from classical physics and the engineering sciences.

1 A typical emergent contextual classical observable is temperature, it is manifest in all global representations which are relevant for the description of systems in thermal equilibrium. 


\section{Exodynamics from endodynamics}

Since maximal symmetry is a typical characteristric of an endophysical first principle, we consider the arrow of time as an exophysical manifestation of a broken time-inversion symmetry, and posit for endophysics a time evolution characterized by a one-parameter group, distinguished by time-inversion symmetry. As a result, in an endophysical codification of quantum mechanics the dynamics is supposed to be time-reflection invariant and bidirectionally deterministic. The concept of probability does not appear in quantum endophysics. That is, quantum endophysics is intrinsically a deterministic nonprobabilistic theory.

On the other hand, quantum exophysics is, by its very nature, a statistical theory which presupposes the existence of statistical Boolean classification devices, hence of external classical measuring systems, which have a nonanticipating Boolean memory, and which show an irreversible dissipative behavior.

Where are the primary probabilities [24] of quantum exophysics coming from? First of all, the endophysical determinism does not imply that the dynamics is fully determinable or predictable by an observer. Any operationalization of the endophysical first principles requires a cut between the observed object and the observing tools. The probabilities of quantum-exophysics enter the picture at the cut between the quantum object and the classical exosystem. Note that primary probabilities are conditional probabilities. The condition is the particular choice of a Heisenberg cut. Although these probabilities are contextual - they depend on our choice of the cut - , they are nevertheless irreducible since the Heisenberg cut cannot be avoided in an operational description. That is, in quantum exophysics, probability is a primary notion, and not - as in classical exophysics - merely a specification of our ignorance. The correctness condition for the statistical interpretation of exophysics is given by the long-run frequencies for theoretical probability statements.

If we describe quantum endophysics by a $\mathrm{C}^{*}$-algebra, and quantum exophysics by a $\mathrm{W}^{*}$-algebra which is induced by a contextually selected topology, we can transfer via the GNS-construction the fundamental endophysical dynamics to the contextual exophysical description. As a rule, such a transition brings about contextual symmetry breakings so that the dynamics of an exophysical description of the endophysical reality can look very different from the basic intrinsic dynamics. For example, there are exactly soluble $\mathrm{C}^{*}$-models with an automorphic (hence time-reflection invariant and bidirectionally deterministic) endophysical dynamics which generate exophysical dynamical semigroups, or even exophysical $\mathrm{W}^{*}$-descriptions with truly irreversible $\mathrm{K}$-flow-type time evolutions. This is not to say that there are no problems in deriving exophysical descriptions from endophysics - but breaking endophysical symmetries is not a mystery (albeit demanding high mathematical skill). 
First problem: Symmetry breaking is not enough

For one thing, every symmetry breaking demands a selection on the part of the observer. For example, if in an exophysical realization the time-inversion symmetry of the dynamics is broken, there exists of necessity a similar but physically different realization with a time arrow in the opposite direction. Additional exophysical regulative principles (e.g. that of Baconian science) are necessary to select an appropriate realization ${ }^{1}$.

\section{Second problem: The endophysical time evolution is not a $C^{*}$-automorphism}

A further, much more serious problem is the fact that as a rule the endophysical dynamics is not given by a one-parameter group of $\mathrm{C}^{*}$-automorphisms ${ }^{2}$. This is not a deathblow for the construction of exophysical dynamical $\mathrm{W}^{*}$-systems but it complicates the mathematical discussion severely since in this case the endophysical $\mathrm{C}^{*}$-dynamics is only well-defined for a dense set of states or observables. On the other hand, this situation is compulsory for the derivation of exophysical nonlinear dynamical semigroups as they are required for the description of classical exosystems. Another, almost paradigmatic example for the mathematical difficulties one encounters in dynamical endo/exo-interface problems is the notorious "measurement problem of quantum mechanics". Unfortunately, most discussions of the measurement process are conceptually and mathematically inadequate, usually based on grossly oversimplified models. It is a simple mathematical fact $^{3}$ that an endophysical automorphic time evolution cannot solve the measurement problem of exophysics.

\section{Endodynamics from exodynamics}

Given a hypothetical $C^{*}$-algebraic quantum endophysics, then the GNS-construction allows us to introduce a contextually selected new topology and to derive an exophysical $\mathrm{W}^{*}$-algebraic description appropriate to the chosen context. The inverse problem of decontextualization, that is, of guessing the universal endophysics from a few exophysical descriptions, is ill-posed. It is therefore quite a miracle how we ever could get reasonable candidates for endophysical laws - certainly they cannot be conceived simply from sense data and empirical regularities without recourse to primordial ideas.

Nonetheless, there are a number of mathematical dilation theorems which possibly may relate Bergsonian time to Newtonian time. The first step is an exophysical systemtheoretical realization of the given phenomenological exophysical description in terms of a new state concept which summarizes the history of the system. This is achieved by a

1 For a more detailed discussion of this problem, compare [31].

2 This statement may irritate mathematicians. Yet, the pre-established harmony between physics and mathematics does not go so far as one could hope for. While the free dynamics for group-theoretically defined elementary systems are usually automorphisms, there is no reason to expect that the introduction of interactions between bare elementary systems leads to automorphic time-evolutions. For an illuminating simple example, compare [8].

3 Compare e.g. [19]. 
transformation of the given mathematically formulated phenomenological evolution law into a state-space description in terms of first-order differential equations. A systemtheoretical state at time $t$ specifies the equivalence class of all histories (for $t<0$ ) of the system which give rise to the same predictions for all conceivable future experiments on the system. Mathematical system theory gives a complete answer to the question of existence, classification and equivalence of state-space realizations. A system-theoretical realization of an exophysical description corresponds to a well-posed Cauchy problem, or equivalently, to a formulation of the given phenomenological law in terms of a dynamical semigroup. Typically, such dynamical semigroups describe irreversible phenomena, and have no inverse elements.

The next, and crucial step in the decontextualization is the passing to a dynamics having a higher symmetry. Since all known candidates for an endophysical dynamical law have a Hamiltonian structure, we may pose a Hamiltonian realization problem: can a given dynamical semigroup be dilated to a Hamiltonian dynamics? There are many results in this direction. For example, every reasonable linear semigroup has a Hamiltonian realization in terms of a strongly continuous one-parameter group of unitary operators acting on some Hilbert space. Such a realization can be considered as a unitary dilation of the systemtheoretical description in terms of an irreversible one-parameter semigroup to a reversible one-parameter group acting in a larger Hilbert space. If such a dilation can be accomplished, Stone's theorem allows to define a selfadjoint generator which in turn gives rise to a Hamiltonian realization in the enlarged Hilbert space. The corresponding unitary operators generate a multiplier $C^{*}$-algebra containing the endophysical $C^{*}$-algebra $\mathscr{}$. A generalization of Stone's theorem for $\mathrm{C}^{*}$-algebras [17] allows then a reconstruction of a corresponding Hamiltonian dynamics on the endophysical level.

A Hamiltonian realization of a genuine damping is always possible but it requires a mechanical model with infinitely many degrees of freedom, even if the original (say Newtonian) description is very simple. The following example is well known. 
Example: Hamiltonian dilation of the simplest semigroup

The exponential $t \mapsto e^{-t / \tau}, t \geq 0,0<\tau<\infty$, is a strictly contractive semigroup of operators in a one-dimensional space. Extending its definition to the negative axis, we obtain the following continuous real-valued function of positive type,

$$
t \mapsto e^{-|t| / \tau}=\frac{1}{\pi} \int_{-\infty}^{\infty} \frac{\tau}{1+(\lambda \tau)^{2}} e^{-i t \lambda} d \lambda
$$

Let $\left\{e^{-i t H} \mid t \in \mathbb{R}\right\}$ be a strongly continuous one-parameter group of unitary operators $e^{-i t H}$, and let $\{E(\lambda)\}$ be the spectral family of the selfadjoint generator $H$,

$$
e^{-i t H}=\int_{-\infty}^{\infty} e^{-i t \lambda} d E(\lambda)
$$

Let furthermore $P$ be a nontrivial projection with one-dimensional range $P \mathscr{H}$. If the measure $P E(\lambda) P$ admits the Radon-Nykodým derivative $(\tau / \pi) /\left\{1+(\lambda \tau)^{2}\right\}$, we can represent the motion $t \mapsto e^{-|t| / \tau}$ by the contracted motion $t \mapsto P e^{-i t H} P$,

$$
e^{-|t| / \tau}=\int_{-\infty}^{\infty} e^{-i t \lambda} d\{P E(\lambda) P\} \quad, \quad \frac{d\{P E(\lambda) P\}}{d \lambda}=\frac{\tau / \pi}{1+(\lambda \tau)^{2}} .
$$

Since the support of the Cauchy distribution $\lambda \mapsto(\tau / \pi) /\left\{1+(\lambda \tau)^{2}\right\}$ is the entire line $\mathbb{R}$, it follows that the spectrum of $H$ is $\mathbb{R}$, simple and absolutely continuous.

The dilation of the semigroup $t \mapsto e^{-t / \tau}$ can be realized in the Hilbert space $L^{2}(\mathbb{R})$ of Lebesgue square integrable functions by

$$
\left\{e^{-i t H} \Phi\right\}(\lambda)=e^{-i t \lambda} \Phi(\lambda), \Phi \in L^{2}(\mathbb{R}),
$$

and the one-dimensional subspace $P \mathscr{H}$ spanned by any normalized squareintegrable functions $\phi$ satisfying

so that

$$
|\phi(\lambda)|^{2}=\frac{\tau / \pi}{1+(\lambda \tau)^{2}}
$$

$$
e^{-t / \tau}=\int_{-\infty}^{\infty} \phi(\lambda)^{*} e^{-i t H} \phi(\lambda) d \lambda
$$

Such mathematical dilation theorems are immensely helpful for guessing endophysical laws, but - of course - they cannot give a recipy to construct endophysics from exophysics. Both, system-theoretical and Hamiltonian dilations are ontologically neutral. A Hamiltonian realization does not claim that it is materially implemented, say by "atoms" or by "infinitely many mechanical degrees of freedom". 


\section{References}

[1] W. Balzer: Introduction. In: Reduction in Science. Ed. by W. Balzer, D. A. Pearce and H. J. Schmidt. Dordrecht. Reidel. 1984. Pp. 1-9.

[2] H. Bergson: Essai sur les données immédiates de la conscience. Paris. 1889. English translation: Time and Free Will: An essay on the Immediate Data of Consciousness. New York. 1910.

[3] N. Bohr: Quantum physics and philosophy, causality and complementarity. In: Philosophy in the MidCentury. A Survey. Ed. by R. Klibansy. Florence. La Nuova Italia Editrice. 1958. Pp. 308-314.

[4] E. du Bois-Reymond: Die Grenzen des Naturerkennens. Leipzig. Veit \& Comp. 1872.

[5] N. Cartwright: How the Laws of Physics Lie. Oxford. Clarendon Press. 1983.

[6] P. Curie: Sur la symétrie dans les phénomènes physiques. Journal de Physique 3, 393 (1894). Reprinted in: Oeuvres de Pierre Curie. Paris. Gauthier-Villars. 1908. Pp. 118-141.

[7] B. d'Espagnat: Open realism. Philosophia Naturalis 28, 54-69 (1991).

[8] M. Fannes and A. Verbeure: On the time evolution automorphisms of the CCR-algebra for quantum mechanics. Commun. Math. Phys. 35, 257-264 (1974).

[9] M. Fierz: Pauli, Wolfgang. In: Dictionary of Scientific Biography. Volume 10. Ed. by C. C. Gillispie. New York. Charles Scribner's Sons. 1970. Pp. 422-425.

[10] D. Finkelstein: Finite physics. In: The Universal Turing Machine. A Half-Century Survey. Ed. by R. Herken. Hamburg. Kammerer \& Unverzagt. 1988. Pp. 349-376.

[11] D. Finkelstein: The universal quantum. In: The World of Contemporary Physics. Ed. by R. F. Kitchener. Albany. State University of New York Press. 1988. Pp. 75-89.

[12] D. Finkelstein: Quantum net dynamics. Int. J. Theor. Phys. 28, 441-467 (1989).

[13] B. C. v. Fraassen: Laws and Symmetry. Oxford. Clarendon Press. 1989.

[14] K. O. Friedrichs: Asymptotic phenomena in mathematical physics. Bull. Amer. Math. Soc. 61, $485-504$ (1955).

[15] W. Heisenberg: Ist eine deterministische Ergänzung der Quantenmechanik möglich? In: Wolfgang Pauli. Wissenschaftlicher Briefwechsel. Band II, 1930-1939. Ed. by K. v. Meyenn. Berlin, 1985. Springer-Verlag. 1935. Pp. 409-418.

[16] W. Heisenberg: Prinzipielle Fragen der modernen Physik. In: Neuere Fortschritte in den exakten Wissenschaften. Fünf Wiener Vorträge. Dritter Zyklus. Leipzig. Franz Deuticke. 1936. Pp. 91-102.

[17] J. Hollevoet, J. Quaegebeur and S. van Keer: Stone's theorem in $C^{*}$-algebras. Quarterly J. Math. 43, 227-233 (1992).

[18] C. G. Jung: The Collected Works of C.G. Jung. Volume 6. Psychological Types. Princeton. Princeton University Press. 1971.

[19] N. P. Landsman: Algebraic theory of superselection sectors and the measurement problem in quantum mechanics. Int. J. Modern Physics 6, 5349-5371 (1991).

[20] J. v. Neumann: Die Eindeutigkeit der Schrödingerschen Operatoren. Mathematische Annalen 104, 570-578 (1931).

[21] J. v. Neumann: Mathematische Grundlagen der Quantenmechanik. Berlin. Springer. 1932.

[22] I. Newton: Opticks. London. Fourth edition, 1730; reprinted by Dover, New York, 1952. 1704.

[23] W. Pauli: Brief vom 19. Februar 1949 an Hermann Levin Goldschmidt. In: Nochmals Dialogik. Ed. by H. L. Goldschmidt. Zürich, 1990. H.L. Goldschmidt, Balgriststrasse 9, CH-8008 Zürich. 1949.

[24] W. Pauli: Wahrscheinlichkeit und Physik. Dialectica 8, 112-124 (1954).

[25] W. Pauli: Phänomen und physikalische Realität. Dialectica 11, 36-48 (1957).

[26] H. Primas: Mathematical and philosophical questions in the theory of open and macroscopic quantum systems. In: Sixty-Two Years of Uncertainty: Historical, Philosophical and Physical Inquiries into the Foundations of Quantum Mechanics. Ed. by A. I. Miller. New York. Plenum. 1990. Pp. 233-257. 
[27] H. Primas: The measurement process in the individual interpretation of quantum mechanics. In: Quantum Theory Without Reduction. Ed. by M. Cini and J. M. Lévy-Leblond. Bristol. Adam Hilger. 1990. Pp. 49-68.

[28] H. Primas: Realistic interpretation of the quantum theory for individual objects. La Nuova Critica 1314, 41-72 (1990).

[29] H. Primas: Zur Quantenmechanik makroskopischer Systeme. In: Wieviele Leben hat Schrödingers Katze? Ed. by J. Audretsch and K. Mainzer. Mannheim. B.I.-Wissenschaftsverlag. 1990.

[30] H. Primas: Necessary and sufficient conditions for an individual description of the measurement process. In: Symposium on the Foundations of Modern Physics 1990. Quantum Theory of Measurement and Related Philosophical Problems. Ed. by P. Lahti and P. Mittelstaedt. Singapore. World Scientific. 1991. Pp. 332-346.

[31] H. Primas: Time-asymmetric phenomena in biology. Complementary exophysical descriptions arising from deterministic quantum endophysics. Open Systems \& Information Dynamics 1, 3-34 (1992).

[32] H. Primas: The Cartesian cut, the Heisenberg cut, and disentangled observers. In: Symposium on the Foundations of Modern Physics 1992. Wolfgang Pauli as a Philosopher. Ed. by K. V. Laurikainen and C. Montonen. Singapore. World Scientific. 1993.

[33] H. Primas: Realism and quantum mechanics. In: Proceedings of the 9th International Congress of Logic, Methodology and Philosophy of Science, Uppsala 1991. Studies in Logic and the Foundations of Mathematics. Ed. by D. Pragwitz, B. Skyrms and D. Westerståhl. Amsterdam. North-Holland. In press.

[34] O. E. Rössler: Endophysics. In: Real Brains, Artificial Minds. Ed. by J. L. Casti and A. Karlqvist. New York. North-Holland. 1987. Pp. 25-46.

[35] O. E. Rössler: Explicit observers. In: Optimal Structures in Heterogeneous Reaction Systems. Springer Series in Synergetics, vol. 44. Ed. by P. J. Plath. Berlin. Springer. 1989. Pp. 123-138.

[36] O. E. Rössler: Boscovich covariance. In: Beyond Belief: Randomness, Prediction and Explanation in Science. Ed. by J. L. Casti and A. Karlqvist. Boca Raton, Ann Arbor, Boston. CRC Press. 1991. Pp. 65-87.

[37] J. D. Sneed: The Logical Structure of Mathematical Physics. Dordrecht. Reidel. 1971.

[38] W. P. v. Stigt: Brouwer's Intuitism. Amsterdam. North Holland. 1990.

[39] M. H. Stone: Linear transformations in Hilbert space. III. Operational methods and group theory. Proc. Nat. Acad. U.S. 16, 172-175 (1930).

[40] M. Takesaki: Theory of Operator Algebras I. New York. Springer. 1979.

[41] G. B. Vico: Prinzipien einer neuen Wissenschaft über die gemeinsame Natur der Völker. Teilband 1 und 2. Übersetzt von V. Hösle und C. Jerman. Mit einer Einleitung von V. Hösle. Hamburg. Meiner. 1990.

[42] H. Weyl: Wissenschaft als symbolische Konstruktion des Menschen. In: Eranos Jahrbuch 1948. Band XVI. Ed. by O. Fröbe-Kapteyn. Zürich. Rhein-Verlag. 1949. Pp. 375-431.

[43] H. Weyl: Über den Symbolismus der Mathematik und mathematischen Physik. Studium generale 6, 219-228 (1953).

[44] H. Weyl: Address on the Unity of Knowledge, delivered at the Bicentennial Conference of Columbia University. In: The Unity of Knowledge. Ed. by L. Leary. New York. Doubleday. 1955.

[45] N. Wiener: Cybernetics, or Control and Communication in the Animal and the Machine. New York. M.I.T. Press and Wiley, second edition 1961. 1948.

[46] C. N. Yang: Magnetic monopoles, fibre bundles, and gauge fields. Annals of New York Academy of Sciences 294, 86-97 (1977). 